\title{
Solid Pseudopapillary Neoplasm of the Pancreas: Unfolding an Intriguing Condition
}

\author{
Manuel António Alves Cruz ${ }^{a}$ Pedro Moutinho-Ribeiro ${ }^{a, b}$ \\ Pedro Costa-Moreira ${ }^{a, b}$ Guilherme Macedo ${ }^{a, b}$ \\ ${ }^{a}$ Faculty of Medicine, University of Porto, Porto, Portugal; ${ }^{b}$ Gastroenterology Department, Centro Hospitalar e \\ Universitário São João, Porto, Portugal
}

Keywords

Pancreas $\cdot$ Neoplasm $\cdot$ Solid pseudopapillary neoplasm

\section{Abstract}

Pancreatic cancer is one of the most lethal malignant neoplasms, with a 1 -year survival rate after diagnosis of $24 \%$, and a 5 -year survival rate of only $9 \%$. While this illustrates the behavior of its main histologic type - ductal adenocarcinoma, there are other histologic subtypes of pancreatic cancer that can harbor excellent prognosis. Solid pseudopapillary neoplasm, described as a rare low-grade malignant neoplasm by the World Health Organization, is the best example of that, having an overall 5 -year survival rate of about $97 \%$. Not only the prognosis, but everything about this entity is unique: its histogenesis, epidemiology, presentation, imaging characteristics, cytology features, immunohistochemical profile, and treatment. This explains the urge to improve our understanding about this entity and thus our ability to accurately recognize and manage it. Having this in mind, this article aims to summarize the most relevant topics regarding this entity.

(c) 2021 Sociedade Portuguesa de Gastrenterologia Published by S. Karger AG, Basel
Neoplasia pseudopapilar sólida do pâncreas: revisão de uma condição intrigante

\section{Palavras Chave}

Pâncreas · Neoplasia · Neoplasia pseudopapilar sólida

\section{Resumo}

O cancro do pâncreas é uma das neoplasias malignas mais letais, com uma taxa de sobrevida 1 ano após o diagnóstico de $24 \%$ e 5 anos após o diagnóstico de apenas $9 \%$. Estes dados espelham, contudo, o comportamento do subtipo histológico mais prevalente - o adenocarcinoma ductal. Porém, nem todas as neoplasias malignas do pâncreas são adenocarcinomas e nem todas estão a associadas a um prognóstico tão reservado. A Neoplasia Pseudopapilar Sólida do Pâncreas é o exemplo disso: descrita pela Organização Mundial da Saúde como uma neoplasia maligna de baixo grau, é um tumor raro associado a um excelente prognóstico, com uma taxa de sobrevida aos 5 anos de $97 \%$. Mais que uma neoplasia com um prognostico peculiarmente favorável, é uma neoplasia única em todas as suas componentes: histogénese, epidemiologia, karger@karger.com www.karger.com/pjg

Karger $\stackrel{\text { ' }}{5}$

BOPEN ACCESS
(C) 2021 Sociedade Portuguesa de Gastrenterologia Published by S. Karger AG, Basel

This is an Open Access article licensed under the Creative Commons Attribution-NonCommercial-4.0 International License (CC BY-NC) (http://www.karger.com/Services/OpenAccessLicense), applicable to the online version of the article only. Usage and distribution for commercial purposes requires written permission.
Correspondence to:

Manuel António Alves Cruz, manelcruzz97@gmail.com 
apresentação clínica, características imagiológicas, citológicas e imunohistoquímicas e no tratamento. Estas particularidades devem estar presentes e consolidadas no raciocínio clínico de qualquer médico, para que estas neoplasias sejam devidamente reconhecidas e tratadas. Neste sentido, foi realizado este artigo de revisão que visa sumariar os mais relevantes tópicos relacionados com esta entidade clínica.

(C) 2021 Sociedade Portuguesa de Gastrenterologia Publicado por S. Karger AG, Basel

\section{Introduction}

Pancreatic cancer is one of the most lethal malignant neoplasms. Despite all recent advances, pancreatic cancer is still diagnosed mostly at advanced stages, with 80 $90 \%$ of the patients presenting unresectable disease at diagnosis, which contributes to a 1-year survival rate after diagnosis of $24 \%$, and a 5 -year survival rate of only $9 \%$ $[1,2]$.

As previously described, pancreatic cancer's natural history mostly illustrates the behavior of its main histologic type - ductal adenocarcinoma, which comprises about $85 \%$ of all pancreatic cancers. However, pancreatic cancer is not always this dark since there are other histologic subtypes that can harbor a favorable prognosis.

Solid pseudopapillary neoplasm (SPN), first described by Frantz in 1959, also known as "Frantz's tumor" [3], is an example. Described as a rare "low-grade malignant pancreatic tumour" by the 2019 WHO Classification of Tumours of the Digestive System [4], SPNs are unique neoplasms, and there is still insufficient information about them in the literature. The few articles that address this topic, do it retrospectively, by means of case series or case reports, making the clinical available knowledge on this issue limited. This article aims to summarize the most relevant topics about this entity.

\section{Methods}

A literature search on PubMed was conducted in January 2021, with backward citation conducted as well. Additionally, two articles were intentionally searched on Google, using, respectively, the queries "Solid Pseudopapillary Neoplasms of the Pancreas Hospital São João" (which represents this institution's experience) and "World Health Organization Classification of Tumors of the Digestive System."
The PubMed search strategy was sensitive to texts and abstracts, using the query: "((solid) AND (pseudopapillary) AND ((neoplasm) OR (tumor))) AND ((pancreas) OR (pancreatic)) AND ((management) OR (Clinical-pathological) OR (Clinicopathological) OR (features)).”

Eighty-four appealing titles and abstracts, considering the scope of the paper, were first selected from the PubMed search's including 596 results. After full text reading, 40 were excluded due to repeated information or no clinical relevance. After searching the references from the 44 studies included, 10 more were added. Finally, the 2 studies from the google search and 2 studies suggested by peer review were also added, totaling 58 articles included in this review.

\section{Tumorigenesis}

SPN is a rare "low-grade malignant pancreatic tumour" (WHO classification 2019) [4] accounting for less than $10 \%$ of the cystic tumors of the pancreas, and up to $1-2 \%$ of all pancreatic tumors [5]. More than rare, it is a unique neoplasm due to its intriguing origin, since its histogenesis still remains obscure and hypothetical owing to the fact that its phenotype does not correlate to any specific lineage: neither pancreatic (either acinar or ductal), nor epithelial, neuroendocrine, or histiocytic. A few hypotheses have been formulated, but there is a recent and apparently more evidence-supported hypothesis stating that this neoplasm is derived from genital-ridge-related cells that were attached to the pancreatic tissue during development. It is supported by the finding that most extra-pancreatic SPNs are not associated with ectopic pancreatic tissue, suggesting a nonpancreatic origin, as well as by the fact that ovarian SPNs are morphologic and genetically similar to pancreatic SPNs [6-8].

From a molecular perspective, SPNs are intriguing as well. Pancreatic ductal adenocarcinoma common genetic alterations (including KRAS, TP53, CDKN2A and SMAD4) are not present in SPNs. Genetic alterations in SPNs are rather related to an aberrant Wnt signaling, which seems to be the hallmark of these neoplasms. In fact, virtually all SPNs are associated with hyperactivation of the Wnt signaling pathway and almost all due to acquired activating mutations of the CTNNB1 oncogene, which result in nuclear $\beta$-catenin accumulation and downregulation of E-cadherin. The latter leads to loss of cohesivity between tumor cells, giving rise to the degenerative changes underlying cystic alterations of SPNs. Other candidate genes implicated include TFE3 and LEF1 [9]. Nonetheless, these genetic alterations are not specific enough to be considered pathognomonic $[6$, 10-12]. 
Table 1. Solid pseudopapillary neoplasm of pancreas: essentials 1

\begin{tabular}{ll}
\hline Epidemiological characteristics & Female predilection (male:female ratio 1:5/9) \\
& 3rd decade of life (males diagnosed at later age) \\
\hline Clinical presentation & Upper abdominal pain or mass \\
& $1 / 3$ asymptomatic and incidentally diagnosed \\
& Rarely metastatic at diagnosis \\
\hline Gross features and localization & Body and tail of the pancreas \\
& Single mass, rarely multicentric \\
& Large size (mean diameter greater than $5 \mathrm{~cm}$ ) \\
& Encapsulated solid mass with cystic component corresponding to hemorrhagic degeneration \\
\hline
\end{tabular}

One of the immunohistochemical hallmarks of this neoplasm is the expression of progesterone receptors (PRs). Considering its higher prevalence among young women and taking into account the larger tumor mean size denoted with higher progesterone serum levels, such as in pregnancy, it could be hypothesized that PRs play a role in tumoral cell replication [13].

\section{Clinical Features}

Because it is a rare disease, most publications are small case series and cannot accurately illustrate the epidemiological behavior of this neoplasm. There are, however, two studies reviewing all SPNs cases documented, on a specific timeline, in the English [14] and both English and Chinese [5] literature. We chose these two publications as models when describing clinicopathological characteristics. In summary, both studies $[5,14]$ agree that patients are predominantly young females (male/female ratio of 1:5.3-9.78), mostly aged between 20 and 30 years old.

A few studies investigated possible differences between SPN in males and females, and a few different clinicopathological features have been shown [15-17]. Most importantly, it seems that males are diagnosed at a later age (Wu et al. [16] reported a peak in incidence at approximately 64 years old) and that these have a poorer prognosis (Table 1).

SPNs can also occur at the pediatric age, but these are otherwise similar to the adult SPNs regarding clinicopathological characteristics [18].

The clinical presentation is nonspecific, as the patients, most frequently, solely complain of upper abdominal pain, bloating discomfort or, less frequently, an upper abdominal mass. Nevertheless, about one-third of the patients are asymptomatic and incidentally diagnosed on imaging. Likewise, no laboratorial abnormality is seen.

Solid Pseudopapillary Neoplasm of the Pancreas
Rare presentations reported include acute rupture of an SPN, which is apparently more common in the pediatric population. It is associated with an acute abdomen presentation that usually follows trauma, or infrequently is spontaneous, possibly mimicking an acute appendicitis or an ovarian-related clinical condition. These conditions are ruled out by surgical exploration or imaging, which will rather show hemoperitoneum and a pancreatic mass, typically of large size (usually greater than $8 \mathrm{~cm}$ in diameter). The importance of this presentation relies on the presumption that this group of patients might have a higher incidence of metastatic disease, which prompts closer and longer follow-up [19].

Other rare presentations include: gastric outlet obstruction [20], splenic vein occlusion, and left-sided extrahepatic portal hypertension associated with gastric varices [21] and recurrent pancreatitis [22].

Despite being mostly a neoplasm of the pancreas, SPNs can also be extra-pancreatic, either derived from ectopic pancreatic tissue or from nonpancreatic tissue. Indeed, there are reports of SPNs located in the mesocolon, greater omentum, retroperitoneum, liver, stomach, duodenum and more recently, and being more frequently recognized as such, in the ovary and testis. These are clinically, histologically, and immunohistochemically similar to the pancreatic counterparts, so that this pathological diagnosis must be taken into consideration when dealing with extra-pancreatic masses that resemble pancreatic SPNs either on imaging or on histology [8, 23-25].

\section{Diagnosis}

Regardless of the clinical manifestations, a diagnosis of SPN is only considered after performing an imaging test, with a preoperative diagnosis obtained with endoscopic ultrasound-guided fine-needle aspiration (EUS-FNA) 
Table 2. Solid pseudopapillary neoplasm of pancreas: essentials 2

Imaging characteristics

Contrast-enhanced CT
Gold standard: better definition of the capsule and excellent characterization of the solid and cystic components, including features such as necrosis, hemorrhage, and calcifications

Generalized weaker early arterial enhancement

Stronger portal-venous phase enhancement in the solid component

Inferior to CT in identifying the capsule and intramural hemorrhage

Spherical and well-demarcated lesion with mix echogenicity, lacking significant blood supply

Isoenhancement during the early phase and hypoenhancement during the late phase

Lesion membrane, intralesional vessel, and intralesional compartmentalization enhancements during arterial phase

MRI

Good for characterization of the cystic component - hemorrhagic degeneration is hyperintense on T1weighted images and has heterogenous signal intensity on T2-weighted images

Signal intensity on T1- and T2-weighted images is heterogeneous

Table 3. Solid pseudopapillary neoplasm of pancreas: essentials 3

Cytohistologic examination
Two randomly mixed components combined with variable amounts of hemorrhage and pseudocystic changes - (1) solid and (2) pseudopapillary

(1) poorly cohesive cells admixed with numerous hyalinized or myxoid fibrovascular cords

(2) discohesive small and monomorphic neoplastic cells that are detached from these fibrovascular stalks

Cercariform cells and nuclear membrane irregularities

Eosinophilic or vacuolated (clear cells) cytoplasm, occasionally containing hyaline globules

Mitoses are uncommon

Vascular and perineural invasion are rare

Immunohistochemical

profile
Positive for $\beta$-catenin (nuclear/cytoplasmatic)

Positive for PR, CD56, vimentin, CD10, and CD99 (paranuclear dot-like pattern)

Aberrant expression of E-cadherin (positivity for antibodies against the cytoplasmic domain tumor but negativity for antibodies against extracellular fragments)

Focally positive for synaptophysin

Negative for chromogranin, BLC10, and trypsin and/or an endoscopic ultrasound-guided fine-needle biopsy (FNB) with immunohistochemical staining (shown in Table 2 and 3) [26].

The neoplasm is most frequently a single mass [27], located in the body and tail of the pancreas $[5,14]$. It is consistently a neoplasm of large size, with a mean diameter of 6 or $8 \mathrm{~cm}$, according to the 2 largest case series available. However, it generally does not invade the surrounding structures nor does it cause biliary ob- struction, even when located in the head of the pancreas [28]. Despite being a low-grade malignant neoplasm, it may rarely present with metastasis, commonly in the liver or infrequently in the peritoneum. When metastasis occur, these develop later in the course of the disease, usually $8-16$ years after primary neoplasm resection $[6,26]$.

Computed tomography (CT) is the gold standard for the detection and evaluation of pancreatic masses, being 

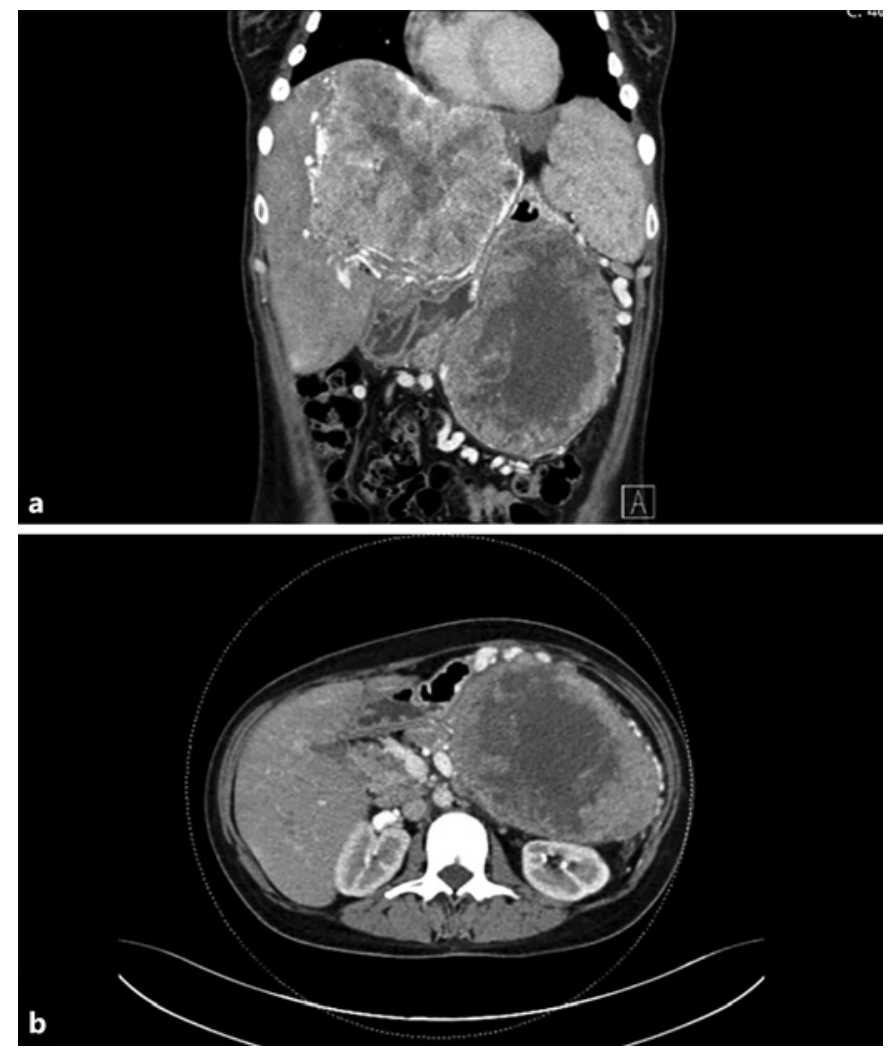

Fig. 1. Contrast-enhanced coronal (a) and axial (b) CT showing a large mass in the tail of the pancreas, with central hypodensity and heterogenous peripherical enhancement, associated with a giant hypervascular hepatic mass (a).

the most frequently performed imaging modality in patients with SPNs $[5,14]$. This neoplasm will appear on CT as a completely or incompletely encapsulated mass, unusually nonencapsulated, with both solid and cystic areas (shown in Fig. 1). Solid areas, sometimes appearing in the form of calcifications, are typically peripherical and correspond to pseudopapillary areas, whereas cystic areas are characteristically central and correspond to hemorrhagic degeneration. Contrast-enhanced CT (CECT) shows generalized weaker early arterial enhancement, but stronger portal-venous phase enhancement in the solid component $[26,29]$.

It has been discussed whether contrast-enhanced endoscopic ultrasonography (CEUS) can offer similar precision in diagnosing SPNs, compared to CECT, since it has several advantages, such as: (1) it provides real-time and dynamic imaging; (2) it does not use radiation; and (3) it can be employed in patients who are allergic to iodinated contrast media or have renal insufficiency [28]. It is becoming consensual that CEUS can, indeed, be a good

Solid Pseudopapillary Neoplasm of the Pancreas
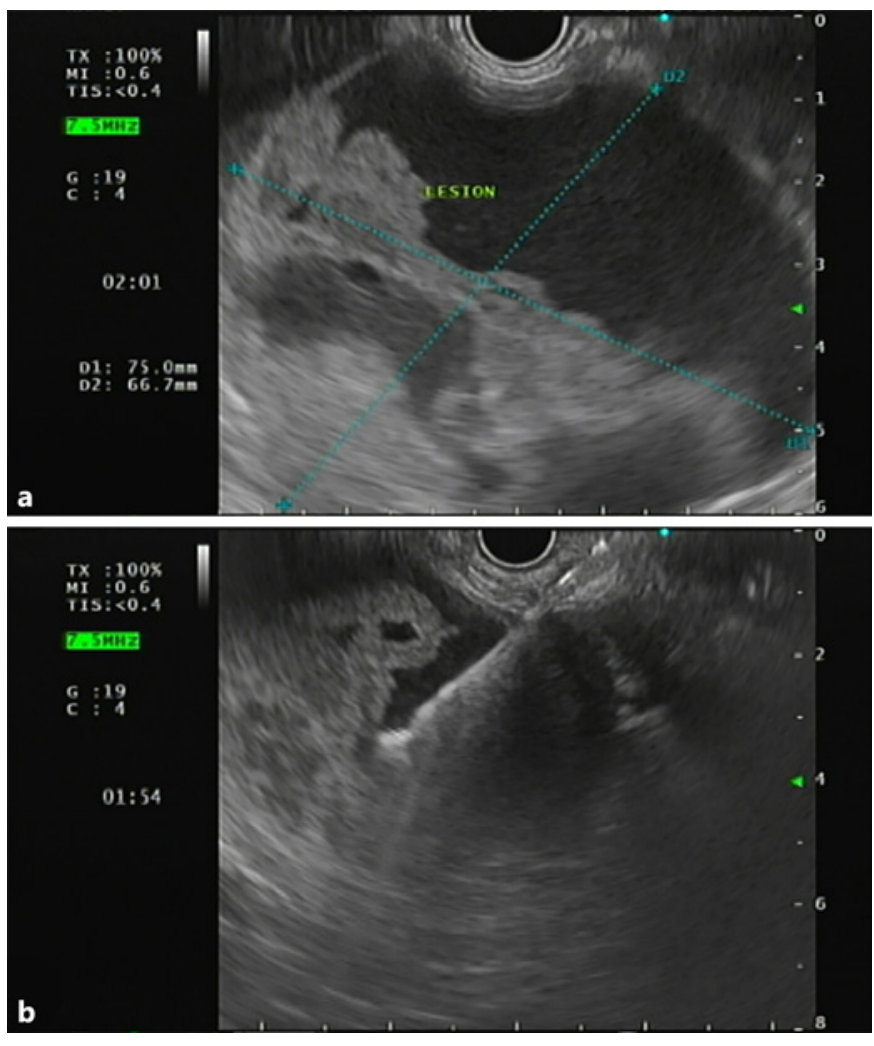

Fig. 2. a Endoscopic ultrasonography showing a large and welldemarcated lesion, located on the body and tail of the pancreas, with mixed echogenicity revealing solid and cystic components. $\mathbf{b}$ Fine-needle aspiration with a 19-gauge needle was performed for cytology and biochemical analysis.

alternative, as it can also show, a suggestive pattern of SPNs: (1) baseline endoscopic ultrasonography (EUS) reveals a spherical and well-demarcated lesion, with mix echogenicity (shown in Fig. 2), lacking significant blood supply; (2) CEUS shows isoenhancement during the early phase and hypoenhancement during the late phase plus lesion membrane, intralesional vessel, and intralesional compartmentalization enhancements during the arterial phase $[26,28,29]$. Nonetheless, CECT is superior to CEUS in identifying the capsule as well as intramural hemorrhage, which are the most important features for diagnosing SPN [26].

Similarly, magnetic resonance imaging (MRI) can be a valuable exam, and can even be considered superior to CECT since, apart from the fact that it lacks radiation and is safer in those who suffer from contrast allergy or renal insufficiency, it better characterizes the cystic component of the lesion. MRI shows an encapsulated lesion with solid and cystic components plus hemorrhage without internal septation (shown in Fig. 3). In addition, the 
signal intensity on T1- and T2-weighted images is heterogeneous, owing to the presence of hemorrhagic degeneration, which is hyperintense on T1-weighted images and has heterogenous signal intensity on T2 weighted images.

Cytohistologic examination associated with immunohistochemistry is mandatory to confirm the diagnosis of SPN [30]. Endoscopic ultrasound-guided fine-needle aspiration (EUS-FA) with the aid of immunocytochemistry on cell block is the most frequently used procedure to accomplish this [31].

Provided that the correct preoperative diagnosis of SPN substantially relies on a cyto-histologic and immunohistochemical analysis, and taking into account the most recent studies comparing the role of FNB versus FNA in the diagnosis of pancreatic masses, EUS-FNB should be considered the gold standard option when tissue acquisition is required to obtain preoperative diagnosis of a SPN, since it provides better specimen adequacy with less needle passes [32-34].

FNA specimens are usually richly cellular, consisting of small discohesive and monomorphic cells surrounded by hemorrhagic debris and eventually multinucleated giant cells and/or foamy histiocytes. These cells display eosinophilic cytoplasm, containing occasionally hyaline globules and/or characteristic nuclear grooves [30].

Histologic examination displays two randomly mixed components combined with variable amounts of hemorrhage and pseudocystic changes: (1) a solid component, composed of poorly cohesive cells admixed with numerous myxoid fibrovascular cords and (2) a pseudopapillary component, corresponding to discohesive small and monomorphic neoplastic cells that are detached from these fibrovascular stalks (shown in Fig. 4) [30]. The neoplastic cells' cytoplasm is either eosinophilic or vacuolated (clear cells) and occasionally contains hyaline globules. Pathologists should look for additional characteristic features that, although not always present, are highly suggestive of SPN, such as cercariform cells $[30,35]$ and nuclear membrane irregularities, particularly nuclear grooves [36]. Mitoses are uncommon and vascular and perineural invasion is rare [30].

Moreover, WHO19 [3] emphasizes an SPN subtype SPN with high-grade carcinoma, describing it as an "SPN with foci of high grade malignant transformation," which is "characterized by diffuse sheets of cells with increased nuclear atypia, as well as abundant mitoses." This subtype is clinically more aggressive [3].

Regarding the immunohistochemical profile, La Rosa and Bongiovanni [30] propose a panel for the rou-
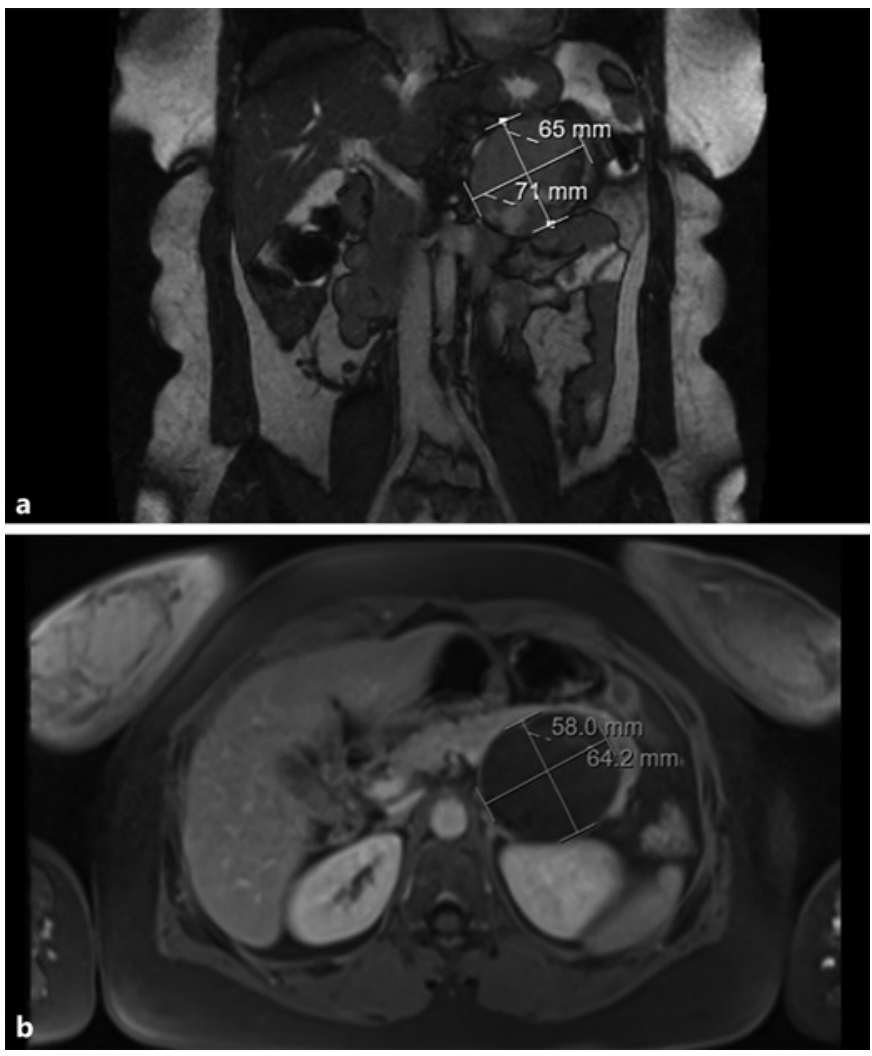

Fig. 3. T2-weighted (a) and T1-weighted (b) magnetic resonance images showing an encapsulated lesion in the body/tail of the pancreas, heterogeneously slightly hyperintense on the T2-weighted image and with low signal intensity on the T1-weighted image. Areas of hemorrhagic degeneration (low signal on the T2- and strong signal on the T1-weighted image) can also be seen.

tine pathology workup of suspected SPN, which includes the following markers: $\beta$-catenin, CD99, chromogranin, trypsin, BCL10, and E-cadherin. SPNs typically show nuclear/cytoplasmic immunoreactivity for $\beta$-catenin, very characteristic immunoreactivity for CD99 (dotlike paranuclear expression) and an aberrant expression of E-cadherin (positivity for antibodies against the cytoplasmic domain tumor but negativity for antibodies against extracellular fragments), while being negative for acinar cell markers (trypsin and BCL10) as well as for neuroendocrine marker chromogranin. Alternatively, we present the panel detailed in the paper by Bouça-Machado et al. [37] that illustrates a case series from our institution: PR (positivity), CD56 (positivity), vimentin (positivity), CD10 (positivity), $\beta$-catenin (nuclear positivity), synaptophysin (focal positivity), al-antitrypsin (focal positivity), and chromogranin (negativity). 

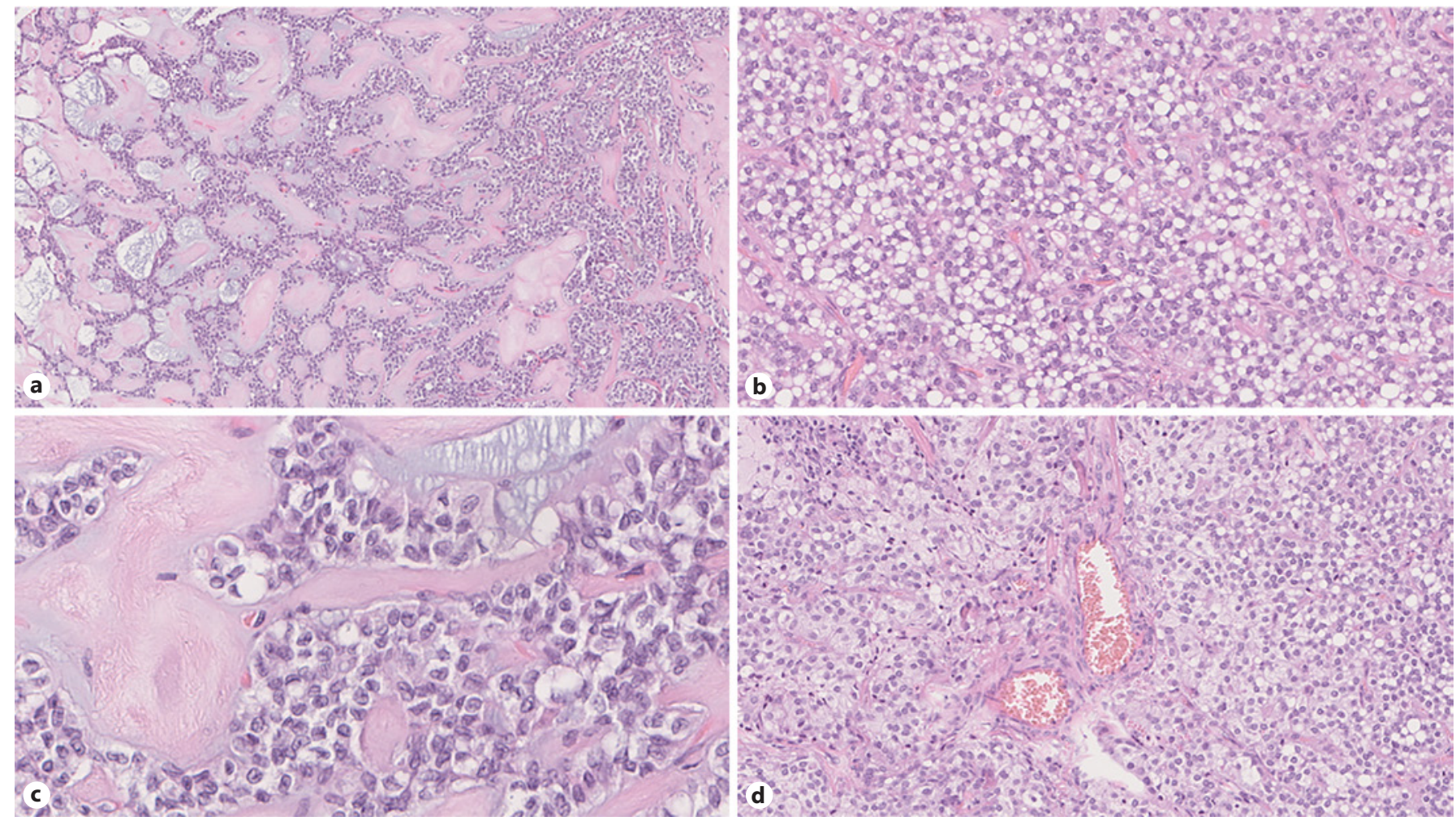

Fig. 4. Histopathology images from surgical specimen showing: a pseudopapillary pattern (hematoxylin and eosin $[\mathrm{HE}]$, original magnification, $\times 13)(\mathbf{a})$; solid tumor tissue featuring cells with clear cytoplasm (HE, original magnification, $\times 30)(\mathbf{b})$; nuclei with grooved membranes $(\mathrm{HE}$, original magnification, $\times 35)(\mathbf{c})$; and hyaline globules between tumor cells (HE, original magnification, $\times 27)(\mathbf{d})$.

\section{Differential Diagnosis}

Main differential diagnosis of SPN include low-grade pancreatic neuroendocrine tumors (pNETs) in adults and pancreatoblastoma $(\mathrm{PB})$ in children $[10,38,39]$. Pseudocyst and other pancreatic cystic neoplasms such as serous cystadenoma (SCA) and mucinous cystic neoplasm (MCN) should also be considered $[9,10,39,40]$.

\section{pNET}

pNET is the major entity when considering differential diagnosis of SPNs in the adult. Nonfunctioning cystic pNETs might clinically behave like SPNs. Despite being typically hypervascular on imaging examination, pNETs have hypovascular forms that can mimic SPNs [41, 42]. Shi et al. [41] have proposed a diagnostic model for differentiation of pNETs and SPNs, based on radiomics scores obtained from MRI's derivations T2-weighted image (T2WI) and diffusion kurtosis imaging (DKI), age, and gender of the patients. Although successfully validated in this study, there is still need for more studies to validate this model, before it can be reliably applicable in clinical practice. Thus, presently the differential diagnosis cannot fully rely on clinical and imaging features. Furthermore, cytohistologic examination might as well be misleading, since a pseudopapillary pattern can resemble pseudorosettes characteristic of pNETs. However, a good cytologic examination will typically show other features consistent with a diagnosis, such as "salt and pepper" chromatin pattern for pNETs or nuclear membrane irregularities (nuclear grooves) for SPNs. Lastly, and more importantly, immunohistochemical examination will clarify and confirm the diagnosis: while pNETs are positive for cytokeratins (CK) 8 and 18 and neuroendocrine marker chromogranin, SPNs are not $[10,40]$. In contrast to SPN, which portrays an excellent prognosis, even if presenting with metastasis or incomplete resection [30] and which is generally treated by neoplasm resection without lymph node dissection nor systemic therapy, pNETs have poorer prognosis (with metastasis: 5-year survival rate of $15-25 \%$; if incomplete resection: survival rate of $35-75 \%$ ) and must be approached more aggressively, with lymph node dissections and systemic therapy if ad- 
Table 4. Solid pseudopapillary neoplasm of pancreas: essentials 4

\begin{tabular}{ll}
\hline Treatment & $\begin{array}{l}\text { Primary surgical resection } \\
\text { Routine lymphadenectomy is controversial }\end{array}$ \\
\hline SPN of the body/tail & Distal pancreatectomy \pm splenectomy, preferably by a laparoscopic approach \\
\hline SPN of the head & Whipple procedure (pancreaticoduodenectomy) \\
\hline Selected cases & Pancreatic enucleation; central pancreatectomy with distal pancreatojejunostomy \\
\hline Metastasis & $\begin{array}{l}\text { Radical metastasectomy, at the time of primary resection; need for adjuvant therapy } \\
\text { is still debatable }\end{array}$ \\
\hline Unresectable primary tumor/metastasis & No recommendation available \\
\hline Prognosis & $\begin{array}{l}5 \text {-year survival rate of 97\% (if resectable) } \\
\text { Adverse prognostic factors }\end{array}$ \\
$\begin{array}{l}\text { Male gender } \\
\text { Positive lymph nodes } \\
\text { R1 margins } \\
\text { Lymphovascular invasion }\end{array}$ \\
\hline
\end{tabular}

vanced locoregional or metastatic disease is present [41]. Thereby, the differential of these entities is crucial to the planning and decision-making processes.

\section{Pancreatoblastoma}

SPNs and PBs are the most frequent neoplasms of the pancreas in children [43]. Despite being clinically identical to SPNs, PBs cytologic examination usually leaves no doubts, since these have a distinctive organoid pattern with characteristic squamoid corpuscles and acinar cells containing zymogen granules [43]. Zhaoxia Yang et al. [38] came up with clinical and imaging features that could be useful in differentiating these entities. Features that support the diagnosis of $\mathrm{PB}$ are age $<5$ years old, elevated alpha-fetoprotein level, not well demarcated tumor margins, size $>6.1 \mathrm{~cm}$, tumoral calcification, peripancreatic vessel invasion, and metastasis at the time of diagnosis. Nevertheless, cytologic examination is still imperative for an accurate preoperative differential diagnosis, which is of great importance since PB is substantially more susceptible to recurrence and metastasis than SPN.

\section{Pseudocyst}

SPNs with extensive cystic degeneration can mimic pseudocysts. However, patient's history, imaging and eventually cytologic and cystic fluid analysis will unveil the diagnosis. Pseudocysts mostly arise following episodes of acute pancreatitis, particularly if superimposed on chronic alcoholic pancreatitis, and are diagnosed following imaging examination showing a thick-walled, rounded and fluid-filled mass in the pancreas. If per- formed, (1) cytologic examination will display debris, blood, inflammatory cells and occasionally yellow pigment or crystals; (2) fluid analysis will have elevated concentration of amylase; and (3) histologic examination will show a pancreatic cystic lesion with no epithelial lining, lined instead by fibrin and granulation tissue.

\section{Other Cystic Neoplasms}

Similar to SPNs, SCAs and MCNs are cystic lesions most often incidentally diagnosed in female patients [40].

- SCA is typically diagnosed in the 6th-7th decade of life and is characterized: (1) on imaging examination, for displaying central, stellate calcifications; (2) on fluid analysis, for having low levels of CEA; (3) histologically for displaying multiple cysts lined by glycogenrich cuboidal cells and cells with round nuclei and remarkably uniform homogenous chromatin; (4) immunohistochemically for staining positive for cytokeratins and negative for CEA [40].

- MCNs are diagnosed almost exclusively in middleaged women and are characterized: (1) on imaging examination, for showing as a multilocular/septated, occasionally with peripheral calcifications neoplasm; (2) on fluid analysis for its thickness and high CEA levels; (3) cytologically for focal clusters of mucin-containing epithelium; (4) histologically for displaying cysts filled with thick mucin and lined by a columnar mucin-producing epithelium associated with a densely cellular ovarian-type stroma; and (5) immunohistochemically for progesterone and estrogen receptor-positive stain in the ovarian stroma [40]. 
still debatable. In the rarer cases of peritoneal metastasis,

Primary surgical resection is the mainstay of treatment for SPNs (shown in Table 4). The procedure of choice will depend on the localization of the tumor. SPN of the body or tail of the pancreas should be resected by distal pancreatectomy, preferably using a laparoscopic approach, with spleen preservation whenever feasible (which can only be considered after exclusion of splenic vasculature and hilar involvement). On the other hand, SPN of the head of the pancreas should be managed by a Whipple procedure (pancreaticoduodenectomy), using an open approach, with pylorus preservation whenever possible, since it reduces the postoperative morbidity by decreasing the risk of dumping syndrome and diarrhea [26, 29, 44].

Since it is a "low grade malignant tumor," when the tumor is localized, there is also the possibility to employ more conservative and parenchyma-preserving techniques, such as pancreatic enucleation and central pancreatectomy with distal pancreatojejunostomy, thus preserving pancreatic exocrine and endocrine functions. Enucleation can be an alternative approach when the tumor is smaller than $2 \mathrm{~cm}$, its distance to the main duct is more than $2-3 \mathrm{~mm}$ and especially when it is localized in the head of the pancreas. Furthermore, it can be conducted laparoscopically. Similarly, central pancreatectomy, by open approach, can be an alternative for tumors localized in the neck of the pancreas and smaller than $3-5 \mathrm{~cm}$. Despite their benefits in maintaining pancreatic functions, these techniques are associated with an increased incidence of pancreatic fistula and a higher postoperative morbidity [45]. Therefore, parenchyma-preserving techniques should be, then, only pursued in young and fit patients who can tolerate the associated higher postoperative morbidity. Also, it could be debatable whether parenchyma-preserving technique indications should not be less strict (for instance, considering safety margins) in the pediatric population [46].

The need for lymphadenectomy in these patients remains a controversial issue: a few authors claim that lymphatic dissections should be employed routinely, but many authors advise against it $[16,26]$. However, when suspicious lymph nodes are found intraoperatively, it is undoubtedly consensual that these should be removed.

In the cases where patients present with liver metastasis, radical metastasectomy, at the time of primary resection, should always be attempted if the lesion is deemed resectable with at least $1 \mathrm{~cm}$ margin. In these situations, generally there is no need for systemic therapy, although the benefits of its use in metastatic resectable tumors are

Solid Pseudopapillary Neoplasm of the Pancreas these can be approached by complete cytoreductive surgery (CCRS) and hyperthermic intraperitoneal chemotherapy (HIPEC) with irinotecan and oxaliplatin $[26,29]$.

If metastatic lesions are not deemed suitable for resection, there is still no consensual recommendation. There are case reports describing successful attempts using adjuvant therapy with: (1) yttrium-90 selective internal radiation therapy (Y-90 SIRT); (2) chemosaturation with percutaneous hepatic perfusions of melphalan; (3) a combination of the tyrosine kinase inhibitor sunitinib and hepatic artery embolization; (4) radiofrequency ablation; (5) liver transplant [26, 29, 47-50].

Lastly, attempts to treat unresectable SPNs with adjuvant chemoradiotherapy (in one report comprising gemcitabine) have been reported in the literature, but there is still lack of evidence to make a recommendation for these situations $[26,29,50]$.

\section{Special Consideration - SPN in Pregnant Women}

SPN diagnosis during pregnancy is rare. Santos et al. [13] summarize all case reports of SPN during pregnancy described in the literature. We have calculated the mean tumor size for these cases $(11.9 \pm 3.6 \mathrm{~cm})$. Although the small population size does not allow statistical inferences with optimal external validity, it seems that SPNs tend to be larger in pregnant women, which could make one think these are more likely to present with symptoms. However, as SPN presentation is highly nonspecific and understandably confoundable with pregnancy common symptoms (abdominal discomfort, nausea, vomiting), diagnosis can be even more challenging. Also, during diagnosis workup, clinicians must think differently when deciding which image modality best suits these patients since the safety of the fetus must be on the top of their priorities. Consequently, modalities without emission of ionizing radiation and without any known adverse fetal effect, such as EUS and MRI, are the gold-standard [13, $51,52]$.

Currently, there is no consensus or guidelines for SPN management in pregnant women. Optimal treatment timing is the core of the discussion, and the only agreement on the matter is that it should be always decided on a case by case basis: clinicians can opt for close surveillance during pregnancy and safely postpone the resection to the postpartum period [52] or, on the other hand, can decide to go ahead with surgical intervention regardless of the gestational age, especially if emergent intervention 
is required (e.g., due to tumor rupture). It is noteworthy that case reports describing surgical intervention during pregnancy showed no harm to fetal development $[13,51$, 52].

\section{Prognosis}

Overall, the prognosis is remarkably good, even in the presence of metastasis, with an overall 5-year survival rate of about $97 \%$. Unresectable disease is associated, on the other hand, with a worse but still fair prognosis, since SPNs are slow-growing neoplasms, with a calculated double time of 765 days [53]. Recurrence after radical resection can occur, though, in $2-10 \%$ of the cases $[5,14,54]$.

The time of follow-up and the conditions requiring closer follow-up are still topics of debate. Nevertheless, an annual surveillance has been suggested for at least 5 years [55].

Several studies have been designed to come up with tumor markers that could predict recurrence or patient outcome. Sex, age, tumor size, surgical margins, perineural invasion, angioinvasion, deep infiltration of surrounding structures, and Ki-67 proliferative index have been suggested, but published results are not consensual [30].

Furthermore, it is noteworthy that the latest systematic review on this topic concluded that features such as male gender (odds ratio [OR] 1.960, 95\% confidence interval [95\% CI] 1.010-3.805, $p=0.047$ ), positive lymph nodes (OR 11.918, 95\% CI 3.798-37.292, p not available), R1 margins (OR 11.132, 95\% CI 4.342-28.541, $p$ not available), and lymphovascular invasion (OR 5.504, 95\% CI 2.461-12.311, $p$ not available) are associated with statistically significant higher odds for recurrence. Thereby, until better consensus, it is advisable to adopt closer follow-up programs in patients presenting with these features [56].

More recently, microRNA expression pattern using a panel of six microRNAs [57] and preoperative neutrophil-to-lymphocyte ratio [58] were suggested to be potential markers, but there is still lack of evidence.

\section{Conclusion}

\section{Key Messages}

- Clinicians should be aware of this entity when approaching a young female presenting with a pancreatic mass.

- Cytohistologic examination associated with immunohistochemistry is required to confirm the diagnosis of SPN.
- EUS-FNB should replace EUS-FNA since it is more accurate for preoperative diagnosis of pancreatic masses.

- Primary surgical resection is the mainstay of treatment for these neoplasms.

- More evidence about the need for lymphadenectomy and the approach to unresectable disease is needed.

- The prognosis is remarkably good, with a 5-year survival rate of about $97 \%$.

- More high-quality studies about poor outcome predictors are needed.

\section{Acknowledgement}

The authors thank Dr. Joanne Lopes for her important contribution, namely in providing the pathological figures and their legends.

\section{Statement of Ethics}

Patient consent statement and permission to reproduce material were obtained.

\section{Conflict of Interest Statement}

The authors have no conflicts of interest to declare.

\section{Funding Sources}

None to report.

\section{Author Contributions}

Manuel Cruz: article concept and design, literature review, and draft of the manuscript.

Pedro Moutinho-Ribeiro: article concept and design and critical review of the manuscript.

Pedro Costa-Moreira, Guilherme Macedo: critical review of the manuscript.

References

1 Rawla P, Sunkara T, Gaduputi V. Epidemiology of Pancreatic Cancer: Global Trends, Etiology and Risk Factors. World J Oncol. 2019 Feb;10(1):10-27.

2 Siegel RL, Miller KD, Fuchs HE, Jemal A. Cancer Statistics, 2021. CA Cancer J Clin. 2021 Jan;71(1):7-33.

3 Frantz VJ. Atlas of tumor pathology, 7th section, 27-28th fascicles. 1959.

Cruz/Moutinho-Ribeiro/Costa-Moreira/ Macedo
GE Port J Gastroenterol 2022;29:151-162 DOI: $10.1159 / 000519933$ 
4 Nagtegaal ID, Odze RD, Klimstra D, Paradis V, Rugge M, Schirmacher P, et al.; WHO Classification of Tumours Editorial Board. The 2019 WHO classification of tumours of the digestive system. Histopathology. 2020 Jan;76(2):182-8.

5 Yao J, Song H. A Review of Clinicopathological Characteristics and Treatment of Solid Pseudopapillary Tumor of the Pancreas with 2450 Cases in Chinese Population. BioMed Res Int. 2020 Jul;2020:2829647.

6 Zalatnai A, Kis-Orha V. Solid-pseudopapillary Neoplasms of the Pancreas is still an Enigma: a Clinicopathological Review. Pathol Oncol Res. 2020 Apr;26(2):641-9.

7 Singh P, Kumar P, Rohilla M, Gupta P, Gupta $\mathrm{N}$, Dey $\mathrm{P}$, et al. Fine needle aspiration cytology with the aid of immunocytochemistry on cell-block confirms the diagnosis of solid pseudopapillary neoplasm of the pancreas. Cytopathology. 2021 Jan;32(1):57-64.

8 Singh K, Patel N, Patil P, Paquette C, Mathews CA, Lawrence WD. Primary Ovarian Solid Pseudopapillary Neoplasm With CTNNB1 c.98C $[\{G T\}] G$ (p.S33C) Point Mutation. Int J Gynecol Pathol. 2018 Mar;37(2):110-6.

9 Deniz K, Arıkan TB, Başkol M, Karahan Öİ. Solid Pseudopapillary Neoplasm of the Pancreas. J Gastrointest Surg. 2021 Jan;25(1):322-4.

10 Dhillon J. Non-Ductal Tumors of the Pancreas. Monogr Clin Cytol. 2020;26:92-108.

11 Jiang Y, Xie J, Wang B, Mu Y, Liu P. TFE3 is a diagnostic marker for solid pseudopapillary neoplasms of the pancreas. Hum Pathol. 2018 Nov;81:166-75.

12 Chetty R, Serra S. Membrane loss and aberrant nuclear localization of E-cadherin are consistent features of solid pseudopapillary tumour of the pancreas. An immunohistochemical study using two antibodies recognizing different domains of the E-cadherin molecule. Histopathology. $2008 \mathrm{Feb} ; 52(3): 325-30$.

13 Santos D, Calhau A, Bacelar F, Vieira J. Solid pseudopapillary neoplasm of pancreas with distant metastasis during pregnancy: a diagnostic and treatment challenge. BMJ Case Rep. 2020 Dec;13(12):e237309.

14 Papavramidis T, Papavramidis S. Solid pseudopapillary tumors of the pancreas: review of 718 patients reported in English literature. J Am Coll Surg. 2005 Jun;200(6):965-72.

15 Zou Y, Huang Y, Hong B, Xiang X, Zhou B, Wei S. Comparison of the clinicopathological features of pancreatic solid pseudopapillary neoplasms between males and females: gender does matter. Histol Histopathol. 2020 Mar;35(3):257-68.

$16 \mathrm{Wu}$ J, Mao Y, Jiang Y, Song Y, Yu P, Sun S, et al. Sex differences in solid pseudopapillary neoplasm of the pancreas: A populationbased study. Cancer Med. 2020 Aug;9(16): 6030-41.

17 Shi S, Zhou Y, Hu C. Clinical manifestations and multi-slice computed tomography characteristics of solid pseudopapillary neoplasms of the pancreas between males and females. BMC Med Imaging. 2019 Nov;19(1):87.
18 Waters AM, Russell RT, Maizlin II, Beierle EA, Dellinger MB, Gow KW, et al.; CCDR Group. Comparison of Pediatric and Adult Solid Pseudopapillary Neoplasms of the Pancreas. J Surg Res. 2019 Oct;242:312-7.

19 Rampersad B, Cave C, Umakanthan S. Rupture of a Solid Pseudopapillary Neoplasm of the pancreas. J Pediatr Surg Case Rep. 2018;30:56-60.

20 McFarlane ME, Plummer JM, Patterson J, Pencle FK. Solid-pseudopapillary tumour of the pancreas as a rare cause of gastric outlet obstruction: a case report. Cases J. 2008 Dec;1(1):374.

21 Nakamura S, Takayama Y, Kuboki Y, Haruyama $\mathrm{H}$, Kishino M, Konishi H, et al. A case of solid pseudopapillary neoplasm of the pancreas presenting with left-sided extrahepatic portal hypertension. Intern Med. 2010;49(16): 1749-53.

22 Belletrutti PJ, Allen PJ, Kurtz RC, DiMaio CJ Education and imaging. Hepatobiliary and pancreatic: recurrent pancreatitis caused by a solid pseudopapillary neoplasm of the pancreas. J Gastroenterol Hepatol. 2011 Apr; 26(4):787.

23 Junzu G, Yanbin S, Suxia W, Janjun D. A case of extrapancreatic solid pseudopapillary tumor in the retroperitoneum. Jpn J Radiol. 2012 Aug;30(7):598-601.

24 Zhu H, Xia D, Wang B, Meng H. Extrapancreatic solid pseudopapillary neoplasm: report of a case of primary retroperitoneal origin and review of the literature. Oncol Lett. 2013 May;5(5):1501-4.

25 Michalova K, Michal M, Sedivcova M, Kazakov DV, Bacchi C, Antic T, et al. Solid pseudopapillary neoplasm (SPN) of the testis: comprehensive mutational analysis of 6 testicular and 8 pancreatic SPNs. Ann Diagn Pathol. 2018 Aug;35:42-7.

26 Lanke G, Ali FS, Lee JH. Clinical update on the management of pseudopapillary tumor of pancreas. World J Gastrointest Endosc. 2018 Sep;10(9):145-55.

27 Yamaguchi M, Fukuda T, Nakahara M, Amano M, Takei D, Kawashima M, et al. Multicentric solid pseudopapillary neoplasms of the pancreas diagnosed by endoscopic ultrasound-guided fine needle aspiration: a case report. Surg Case Rep. 2015 Dec;1(1):110.

28 Xu M, Li XJ, Zhang XE, Pan FS, Tan Y, Huang TY, et al. Application of Contrast-Enhanced Ultrasound in the Diagnosis of Solid Pseudopapillary Tumors of the Pancreas: Imaging Findings Compared With Contrast-Enhanced Computed Tomography. J Ultrasound Med. 2019 Dec;38(12):3247-55.

29 Gandhi D, Sharma P, Parashar K, Kochar PS, Ahuja K, Sawhney H, et al. Solid pseudopapillary Tumor of the Pancreas: radiological and surgical review. Clin Imaging. 2020 Nov;67:101-7.

30 La Rosa S, Bongiovanni M. Pancreatic Solid Pseudopapillary Neoplasm: Key Pathologic and Genetic Features. Arch Pathol Lab Med. 2020 Jul;144(7):829-37.
31 Ardengh JC, Lopes CV, Venco FE, Machado MA. Diagnosis of pancreatic solid pseudopapillary neoplasms using cell-blocks and immunohistochemical evaluation of endoscopic ultrasound-guided fine needle aspiration biopsy specimens. Cytopathology. 2021 Jan;32(1):50-56.

32 Mangiavillano B, Sosa-Valencia L, Deprez P, Eisendrath P, Robles-Medranda C, Eusebi $\mathrm{LH}$, et al. Tissue acquisition and pancreatic masses: which needle and which acquisition technique should be used? Endosc Int Open. 2020 Oct;8(10):E1315-20.

33 Khoury T, Sbeit W, Ludvik N, Nadella D, Wiles A, Marshall C, et al. Concise review on the comparative efficacy of endoscopic ultrasound-guided fine-needle aspiration vs core biopsy in pancreatic masses, upper and lower gastrointestinal submucosal tumors. World J Gastrointest Endosc. 2018 Oct;10(10):267-73.

34 Cheng B, Zhang Y, Chen Q, Sun B, Deng Z, Shan H, et al. Analysis of Fine-Needle Biopsy vs Fine-Needle Aspiration in Diagnosis of Pancreatic and Abdominal Masses: A Prospective, Multicenter, Randomized Controlled Trial. Clin Gastroenterol Hepatol. 2018 Aug;16(8):1314-21.

35 Samad A, Shah AA, Stelow EB, Alsharif M, Cameron SE, Pambuccian SE. Cercariform cells: another cytologic feature distinguishing solid pseudopapillary neoplasms from pancreatic endocrine neoplasms and acinar cell carcinomas in endoscopic ultrasound-guided fine-needle aspirates. Cancer Cytopathol. 2013 Jun;121(6):298-310.

36 Misra S, Saran RK, Srivastava S, Barman S, Dahale A. Utility of cytomorphology in distinguishing solid pseudopapillary neoplasm of pancreas from pancreatic neuroendocrine tumor with emphasis on nuclear folds and nuclear grooves. Diagn Cytopathol. 2019 Jun;47(6):531-40.

37 Bouça-Machado T, Bessa-Melo R, Lopes J, Graca L, Costa-Maia J. Solid Pseudopapillary Neoplasm of the Pancreas: Experience of a Tertiary Hospital. Surg Gastroenterol Oncol. 2020;25(1):30.

38 Yang Z, Gong Y, Ji M, Yang B, Qiao Z. Differential diagnosis of pancreatoblastoma $(\mathrm{PB})$ and solid pseudopapillary neoplasms (SPNs) in children by CT and MR imaging. Eur Radiol. 2021 Apr;31(4):2209-2217.

39 Ray Choudhury S, Mohanty S, Mohapatra D, Sahoo N, Panda A. An Atypical Presentation of Pancreatic Pseudocyst Masquerading as Solid Pseudopapillary Neoplasm of Pancreas. Cureus. 2020 Aug;12(8):e9883.

40 Abdelkader A, Hunt B, Hartley CP, Panarelli NC, Giorgadze T. Cystic Lesions of the Pancreas: Differential Diagnosis and CytologicHistologic Correlation. Arch Pathol Lab Med. 2020 Jan;144(1):47-61.

41 Shi YJ, Zhu HT, Liu YL, Wei YY, Qin XB, Zhang XY, et al. Radiomics Analysis Based on Diffusion Kurtosis Imaging and T2 Weighted Imaging for Differentiation of Pancreatic Neuroendocrine Tumors From Solid Pseudopapillary Tumors. Front Oncol. 2020 Aug;10:1624.
Solid Pseudopapillary Neoplasm of the Pancreas
GE Port J Gastroenterol 2022;29:151-162 DOI: $10.1159 / 000519933$ 
42 Wang C, Cui W, Wang J, Chen X, Tong H, Wang Z. Differentiation between solid pseudopapillary neoplasm of the pancreas and hypovascular pancreatic neuroendocrine tumors by using computed tomography. Acta Radiol. 2019 Oct;60(10):1216-23.

43 Dhebri AR, Connor S, Campbell F, Ghaneh P, Sutton R, Neoptolemos JP, et al. Diagnosis, treatment and outcome of pancreatoblastoma. Pancreatology. 2004;4(5):441-51.

44 Namur GN, Ribeiro TC, Souto MM, Figueira ER, Bacchella T, Jureidini R. MINIMALLY INVASIVE SURGERY FOR PSEUDOPAPILLARY NEOPLASM OF THE PANCREAS. Arq Bras Cir Dig. 2016 Apr-Jun;29(2):97-101.

45 Gharios J, Hain E, Dohan A, Prat F, Terris B, Bertherat J, et al. Pre- and intraoperative diagnostic requirements, benefits and risks of minimally invasive and robotic surgery for neuroendocrine tumors of the pancreas. Best Pract Res Clin Endocrinol Metab. 2019 Oct;33(5):101294.

46 Yalçın B, Yağcı-Küpeli B, Ekinci S, Orhan D, Oğuz B, Varan A, et al. Solid pseudopapillary neoplasm of the pancreas in children: hacettepe experience. ANZ J Surg. 2019 Jun;89(6): E236-40.

47 Dyas AR, Johnson DT, Rubin E, Schulick RD, Kumar Sharma P. Yttrium-90 selective internal radiotherapy as bridge to curative hepatectomy for recurrent malignant solid pseudopapillary neoplasm of pancreas: case report and review of literature. J Surg Case Rep. 2020 Sep;2020(9):rjaa325. https://doi.org/10.1093/ jscr/rjaa325.
48 Escobar MA Jr, McClellan JM, Thomas W. Solid pseudopapillary tumour (Frantz's tumour) of the pancreas in childhood: successful management of late liver metastases with sunitinib and chemoembolisation. BMJ Case Rep. 2017 Dec;2017:bcr2017221906.

49 Hofmann H, von Haken R, Werner J, Kortes $\mathrm{N}$, Bergmann F, Schemmer P, et al. Unresectable isolated hepatic metastases from solid pseudopapillary neoplasm of the pancreas: a case report of chemosaturation with highdose melphalan. Pancreatology. 2014 NovDec;14(6):546-9.

50 Maffuz A, Bustamante FT, Silva JA, TorresVargas S. Preoperative gemcitabine for unresectable, solid pseudopapillary tumour of the pancreas. Lancet Oncol. 2005 Mar;6(3):1856

51 Huang TT, Zhu J, Zhou H, Zhao AM. Solid pseudopapillary neoplasm of pancreas in pregnancy treated with tumor enucleation: Case report and review of the literature. Niger J Clin Pract. 2018 Sep;21(9):1234-1237.

52 Yee AM, Kelly BG, Gonzalez-Velez JM, Nakakura EK. Solid pseudopapillary neoplasm of the pancreas head in a pregnant woman: safe pancreaticoduodenectomy postpartum. J Surg Case Rep. 2015 Aug;2015(8):rjv108. https://doi.org/10.1093/jscr/rjv108.
53 Kato T, Egawa N, Kamisawa T, Tu Y, Sanaka M, Sakaki N, et al. A case of solid pseudopapillary neoplasm of the pancreas and tumor doubling time. Pancreatology. 2002;2(5):4958.

54 Liu M, Liu J, Hu Q, Xu W, Liu W, Zhang Z, et al. Management of solid pseudopapillary neoplasms of pancreas: A single center experience of 243 consecutive patients. Pancreatology. 2019 Jul;19(5):681-5.

55 Gerry JM, Poultsides GA. Surgical Management of Pancreatic Cysts: A Shifting Paradigm Toward Selective Resection. Dig Dis Sci. 2017 Jul;62(7):1816-26.

56 Yepuri N, Naous R, Meier AH, Cooney RN, Kittur D, Are C, et al. A systematic review and meta-analysis of predictors of recurrence in patients with Solid Pseudopapillary Tumors of the Pancreas. HPB (Oxford). 2020 Jan;22(1):12-9.

57 Cohen SJ, Papoulas M, Graubardt N, Ovdat E, Loewenstein S, Kania-Almog J, et al. MicroRNA Expression Patterns Predict Metastatic Spread in Solid Pseudopapillary Neoplasms of the Pancreas. Front Oncol.2020 Mar;10:328.

58 Yang F, Bao Y, Zhou Z, Jin C, Fu D. Preoperative neutrophil-to-lymphocyte ratio predicts malignancy and recurrence-free survival of solid pseudopapillary tumor of the pancreas. J Surg Oncol. 2019 Aug;120(2):241-8. 